\title{
ESTIMATION OF THE CONVECTIVE HEAT TRANSFER COEFFICIENT IN PIPELINES WITH THE MARKOV CHAIN MONTE CARLO METHOD
}

\author{
L.A.B. Varón ${ }^{1}$, H.R.B. Orlande ${ }^{1}$, F.L.V. Vianna ${ }^{2}$
}

${ }^{1}$ Politécnica/COPPE, Department of Mechanical Engineering, Federal University of Rio de Janeiro (lebermeo@gmail.com, helcio@mecanica.ufrj.br)

${ }^{2}$ Department of Subsea Technology, Petrobras Research and Development Center - CENPES (fvianna@ petrobras.com.br)

\begin{abstract}
The flow of hydrocarbons through deep sea pipelines is a challenging issue for the petroleum industry. Typical operating conditions involve high hydrostatic pressures and low sea bed temperatures, which can favor the formation of solid deposits and result in pipeline blockages and, consequently, incur in large financial losses. Heat transfer analysis plays a fundamental role in the design of deep sea pipelines. Thermal insulation is designed to avoid the formation of solid deposits during regular operating conditions. On the other hand, during shutdown periods heat losses from the produced fluid to the surrounding environment can result on fluid temperatures sufficiently low that the formation of deposits becomes inevitable, unless other techniques are used, such as injection of chemical inhibitors or active heating of the pipeline. In this work, we solve the inverse problem of estimating the transient heat transfer coefficient from the pipeline surface to the surrounding sea water, in a pipe-in-pipe system. The transient external heat transfer coefficient is estimated with the Markov Chain Monte Carlo method, implemented via the Metropolis-Hastings algorithm. Simulated temperature measurements of one single sensor, located at the external surface of the inner pipe, are used in the inverse analysis. A smooth prior is used for the transient heat transfer coefficient, while the measurement errors are assumed to be Gaussian, additive, with zero mean and known covariance matrix.
\end{abstract}

Keywords: pipelines, Markov Chain Monte Carlo, convection coefficient, direct problem, inverse problem.

\section{INTRODUCTION}

Oil flow through pipelines in deep sea waters is one of the greatest challenges for the development of subsea field layouts, being characterized by numerous technical problems related to the dynamic nature of the production fluids [1].The thermal performance of such subsea systems is critical, because the produced fluid cannot undergo significant temperature reductions as it flows through the pipeline at high pressures. Therefore, flow assurance is a 
key point for the design of subsea petroleum systems in deep sea [2], involving the prediction and prevention of paraffinic wax deposits and hydrate formations, which can interrupt the flow and cause large financial losses [3].

The main objective in this work is to estimate the transient heat transfer coefficient from the pipeline surface to the surrounding sea water, in a pipe-in-pipe system. The physical problem under study involves a typical pipeline cross-section during a production shutdown period. It is represented by a circular domain filled by the stagnant petroleum fluid, which is bounded by a multilayered wall. The transient external heat transfer coefficient is estimated with the Markov Chain Monte Carlo method, implemented via the Metropolis-Hastings algorithm [4-15]. Simulated temperature measurements of one single sensor, located at the external surface of the inner pipe, are used in the inverse analysis. A smooth prior is used for the transient heat transfer coefficient, while the measurement errors are assumed to be Gaussian, additive, with zero mean and known covariance matrix.

\section{PHYSICAL PROBLEM AND MATHEMATICAL FORMULATION}

The problem considered in this work consists of the cross-section of a pipeline (pipein-pipe), represented by a circular domain filled with a stagnant fluid [1,2]. The pipe-in-pipe system consists of two concentric steel pipes, with a thermal isolation in the annular space between them. A temperature sensor is located outside the inner pipe wall, as shown in Figure 1.

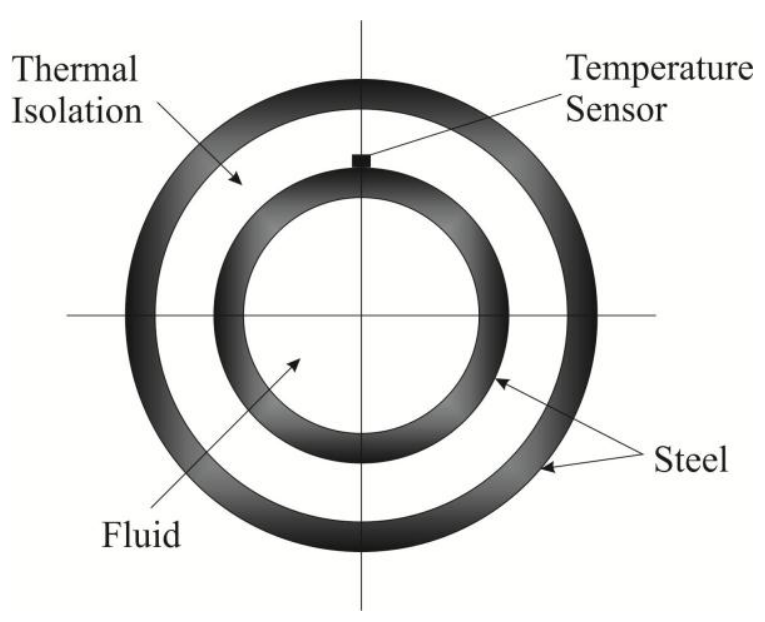

Figure 1.Cross-Section of Pipeline

The fluid and the other materials are considered as homogeneous, isotropic and with constant thermophysical properties. Heat transfer in the pipe-in-pipe system is formulated in terms of a linear transient heat conduction problem in a medium with spatially variable thermophysical properties. Convective heat losses between the surface of the pipe-in-pipe and the surrounding sea water takes place with a time varying heat transfer coefficient. By considering axial symmetry, the formulation of this heat conduction problem in cylindrical coordinates and in dimensionless form is given by: 


$$
\begin{array}{ll}
C^{*}(R) \frac{\partial \theta(R, \tau)}{\partial \tau}=\frac{1}{R} \frac{\partial}{\partial R}\left(k^{*}(R) R \frac{\partial \theta(R, \tau)}{\partial R}\right) & 0 \leq R<1, \tau>0 \\
k^{*}(R) \frac{\partial T}{\partial R}+B i(\tau) \theta(R, \tau)=0 & R=1, \tau>0 \\
\theta(R, 0)=1 & 0 \leq R<1, \tau=0
\end{array}
$$

where the following dimensionless variables were defined:

$$
\begin{gathered}
C^{*}(R)=\frac{C(r)}{C_{r e f}} \\
k^{*}(R)=\frac{k(r)}{k_{r e f}} \\
R=\frac{r}{r^{*}} \\
\tau=\frac{k_{r e f} t}{C_{r e f} r^{*^{2}}} \\
\theta(R, \tau)=\frac{T(r, t)-T_{\infty}}{T(r, 0)-T_{\infty}} \\
B i(\tau)=\frac{h(t) r^{*}}{k_{r e f}}
\end{gathered}
$$

In equations (4-9), $r^{*}$ is the external radius of the pipe-in-pipe, $\mathrm{h}(\mathrm{t})$ is the convective heat transfer coefficient at the outer surface of the pipe-in-pipe, $C^{*}$ and $k^{*}$ are the volumetric heat capacity and thermal conductivity, respectively, $\mathrm{T}_{\infty}$ is the surrounding environment temperature, $\mathrm{k}_{\text {ref }}$ and $\mathrm{C}_{\text {ref }}$ are reference values for the volumetric heat capacity and thermal conductivity, respectively, which are taken as those of the steel pipes.

For the direct problem associated with equations (1-9), initial and boundary conditions, as well as the thermophysical properties are known. The objective of the direct problem is to compute the transient temperature field $\theta(R, \tau)$. The solution of the direct problem was obtained here with the Matlab's function PDEPE.

This work deals with the solution of an inverse problem aiming at estimating the transient heat transfer coefficient, in dimensionless form represented by the Biot number $\operatorname{Bi}(\tau)$, by using transient temperature measurements taken at the external surface of the inner pipe, as illustrated by Figure 1.

For the solution of the inverse problem, the transient heat transfer coefficient is discretized in terms of constant values $\mathbf{P}_{i}=B i\left(\tau_{l}\right)$ in the time intervals $\tau_{i-1} \leq \tau<\tau_{i}$, for $i=1, \ldots, I$, and conveniently written in terms of a vector

$$
\mathbf{P}^{T} \equiv\left[P_{1}, P_{2}, \ldots, P_{I}\right]
$$

The transient temperature measurements of the single sensor are also suitable arranged in the following vector

$$
\mathbf{Y}^{T}=\left(Y_{1}, Y_{2}, \ldots, Y_{I}\right)
$$


The solution of the present inverse problem is obtained within the Bayesian framework, as described next.

\section{MARKOV CHAIN MONTE CARLO METHOD}

The solution of the inverse problem within the Bayesian framework is recast in the form of statistical inference from the posterior probability density, which is the model for the conditional probability distribution of the unknown parameters given the measurements. The measurement model incorporating the related uncertainties is called the likelihood, that is, the conditional probability of the measurements given the unknown parameters. The model for the unknowns that reflects all the uncertainty of the parameters without the information conveyed by the measurements is called the prior model [4-11].

The Bayesian statistics consists mainly in the use of all information available in order to reduce the uncertainty on inference or decision making problems. In the Bayes' theorem, as new information is available it is combined with the previous one forming the basis of the statistical process [4-10]. The inverse problem solution with Bayes' theorem is based in the following principles [11]:

- All variables included in the model are modeled as random variables.

- The randomness describes the degree of information concerning their realizations.

- The degree of information concerning these values is coded in probability distributions.

- The solution of the inverse problem is the posterior probability distribution, from which distribution point estimates and other statistics are computed.

Therefore, this approach relies fundamentally on the principles of the Bayesian statistics to obtain the solution of inverse problems. [12]. Bayes' theorem is stated as:

$$
\pi_{\text {posterior }}(\mathbf{P})=\pi(\mathbf{P} \mid \mathbf{Y})=\frac{\pi(\mathbf{P}) \pi(\mathbf{Y} \mid \mathbf{P})}{\pi(\mathbf{Y})}
$$

where $\pi_{\text {posterior }}(\mathbf{P})$ is de posterior probability density, $\pi(\mathbf{P})$ is the prior probability density, $\pi(\mathbf{Y} \mid \mathbf{P})$ is the likelihood function and $\pi(\mathbf{Y})$ is the marginal probability density of the measurements $\mathbf{Y}$, which plays the role of a normalizing constant.

The Markov Chain Monte Carlo (MCMC) method is used for the estimation of the posterior probability density, which is constructed through sampling and rejection techniques. The most common algorithm of MCMC mthods is the one due to Metropolis-Hastings [5-11]. The implementation of the Metropolis-Hastings algorithm starts with the selection of a jumping distribution $q\left(\mathbf{P}^{*}, \mathbf{P}^{t-1}\right)$ which is used to draw a new candidate state $\mathbf{P}^{*}$, given the current state $\mathbf{P}^{t-1}$ of the Markov chain. Once the jumping distribution has been selected, the Metropolis-Hastings sampling algorithm can be implemented as shown in Table 1 [12]: 
Table 1.Metropolis Hasting Algorithm

1. Sample a Candidate Point $\mathbf{P}^{*}$ from a jumping distribution $q\left(\mathbf{P}^{*}, \mathbf{P}^{t-1}\right)$.

2. Calculate the acceptance factor.

$$
\alpha=\min \left[1, \frac{\pi\left(\mathbf{P}^{*} \mid \mathbf{Y}\right) q\left(\mathbf{P}^{t-1}, \mathbf{P}^{*}\right)}{\pi\left(\mathbf{P}^{t-1} \mid \mathbf{Y}\right) q\left(\mathbf{P}^{*}, \mathbf{P}^{t-1}\right)}\right]
$$

3. Generate the random value $U$ with uniform distribution between 0 e 1 .

4. If $U \leq \alpha$, set $\mathbf{P}^{t}=\mathbf{P}^{*}$. Otherwise, set $\mathbf{P}^{t}=\mathbf{P}^{t-1}$

5. Return to step 1 in order to generate the sequence $\left\{\mathbf{P}^{1}, \mathbf{P}^{2}, \ldots, \mathbf{P}^{n}\right\}$.

In this way, a sequence is generated to represent the posterior distribution and inference on this distribution is obtained from inference on the samples $\left\{\mathbf{P}^{1}, \mathbf{P}^{2}, \ldots, \mathbf{P}^{n}\right\}$. The values of $\mathbf{P}^{i}$ must be ignored until the chain has not converged to equilibrium (the burn-in period) $[14,15]$.

\section{RESULTS AND DISCUSSIONS}

For the test cases presented below, we consider the pipe-in-pipe (see Figure 1) to be made of an inner steel pipe with internal and external diameters of $0.2 \mathrm{~m}$ and $0.25 \mathrm{~m}$, respectively. The outer pipe is also made of steel with internal and external diameters of $0.35 \mathrm{~m}$ and $0.4 \mathrm{~m}$, respectively. The thermophysical properties of steel are assumed as constant and given by [15]: $k=52.34 \mathrm{~W} / \mathrm{m}^{\circ} \mathrm{C}, \rho=7700 \mathrm{~kg} / \mathrm{m}^{3}$ and $c_{p}=502.1 \mathrm{~J} / \mathrm{kg}^{\circ} \mathrm{C}$. The annular space between the two pipes is assumed to be filled with a thermal insulator with constant thermophysical properties given by [1]: $k=0.17 \mathrm{~W} / \mathrm{m}^{\circ} \mathrm{C}, \rho=750 \mathrm{~kg} / \mathrm{m}^{3}$ and $c_{p}=2000 \mathrm{~J} / \mathrm{kg}^{\circ} \mathrm{C}$, which approximates those for polypropylene. For the petroleum, the physical properties are $k=$ $0.1304 \mathrm{~W} / \mathrm{m}^{\circ} \mathrm{C}, \rho=966.7971 \mathrm{~kg} / \mathrm{m}^{3}$ and $c_{p}=1591.7178 \mathrm{~J} / \mathrm{kg}^{\circ} \mathrm{C}$.

By assuming that the measurement errors are Gaussian random variables, with zero means and known covariance matrix $\mathbf{W}$ and that the measurement errors are additive and independent of the parameters $\mathbf{P}$, the likelihood function can be expressed as [4-15]:

$$
\pi(\mathbf{Y} \mid \mathbf{P})=(2 \pi)^{-I / 2}|\mathbf{W}|^{-1 / 2} \exp \left\{-\frac{1}{2}[\mathbf{Y}-\boldsymbol{\Theta}(\mathbf{P})]^{T} \mathbf{W}^{-1}[\mathbf{Y}-\boldsymbol{\Theta}(\mathbf{P})]\right\}
$$

where $\Theta(\mathbf{P})$ is the solution of the direct (forward) problem given by equations (1-3), obtained with a sample of the parameter vector $\mathbf{P}$.

A smoothness prior [5] is used for the Biot number in this work. This prior is appropriate for problems such as the one under consideration because, at the same time that it is non-informative and do not impose restrictions on the marginal distributions of Biot number components, it results on stable solutions for the inverse problem. The smoothness prior used in this work is given by:

$$
\pi(\mathbf{P}) \propto \exp \left(-\frac{1}{2} \alpha \sqrt{\mathbf{P}^{T} \mathbf{Z P}}\right)
$$

where $\mathbf{Z}=\mathbf{D}^{\mathrm{T}} \mathbf{D}$ and $\alpha^{-1}$ is a parameter associated to the variance of $\mathbf{P}$, while $\mathbf{D}$ is a (I-1) $\mathbf{X} \mathbf{I}$ first-order difference matrix given by: 


$$
\mathbf{D}=\left[\begin{array}{ccccc}
-1 & 1 & 0 & \ldots & 0 \\
0 & -1 & 1 & \ldots & 0 \\
0 & \ldots & 0 & -1 & 1
\end{array}\right]
$$

The parameter $\alpha$ appearing in the smoothness priors is treated in this work as a hyper parameter, that is, it is estimated as part of the inference problem in a hierarchical model [5]. The hyperprior density for this parameter is taken in the form of a Rayleigh distribution, with a center $\alpha_{0}=300$. Therefore, the posterior distribution is given by:

$$
\pi(\alpha, \mathbf{P} \mid \mathbf{Y}) \propto \alpha \exp \left\{-\frac{1}{2}[\mathbf{Y}-\mathbf{\Theta}(\mathbf{P})]^{T} \mathbf{W}^{-1}[\mathbf{Y}-\boldsymbol{\Theta}(\mathbf{P})]-\frac{1}{2} \alpha \sqrt{\mathbf{P}^{T} \mathbf{Z P}}-\frac{1}{2}\left(\frac{\alpha}{\alpha_{0}}\right)^{2}\right\}
$$

The test cases examined below involved typical conditions resulting from a shutdown in the petroleum flow through the pipeline system, where the stagnant fluid was assumed to be initially at the uniform temperature of $80^{\circ} \mathrm{C}$. The initial states within the steel pipes and the thermal insulator were given by the steady-state temperature distribution resulting from the heat losses through the pipe-in-pipe multilayered wall. The seawater was assumed to be at $4^{\circ} \mathrm{C}$. The inverse problem was focused on the cooling period of the production fluid, during a production shutdown. The evolution of the dimensionless temperature field in the pipeline system, for the conditions presented above, is shown by the contour plots in Figure 2, for a dimensionless time $\tau=13.8$.

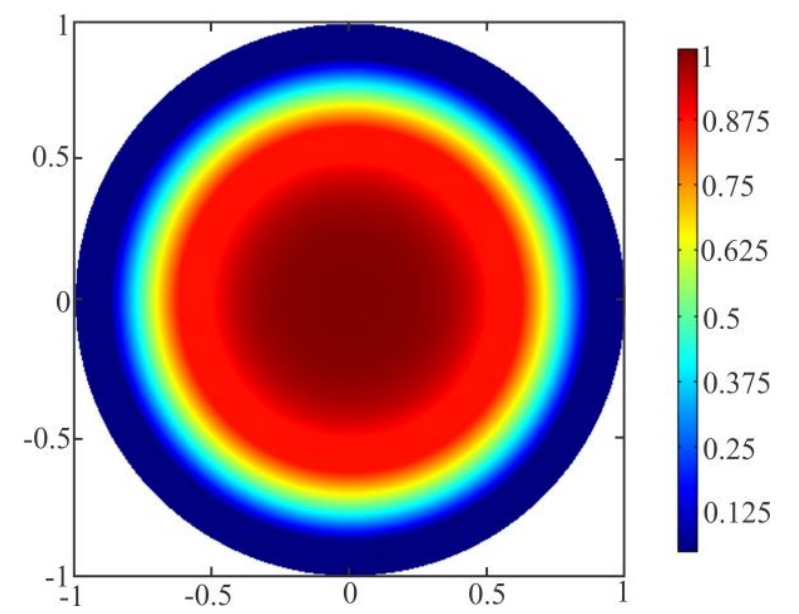

Figure 2. Dimensionless Temperature Distribution for $\tau=13.8$

The accuracy of the present inverse problem solution approach was examined by using simulated transient measurements. The simulated measurements contained random errors which were assumed to be additive, Gaussian, uncorrelated, with zero mean and constant standard deviation. Figure 3 presents the exact temperatures and the simulated measurements for a standard deviation $\sigma=0.01 \boldsymbol{\theta}_{\max }$. The maximum temperature in the system is the initial temperature of the stagnant fluid which is of $80^{\circ} \mathrm{C}$. Therefore, in this case the standard deviation of the measurement errors corresponds to $0.8^{\circ} \mathrm{C}$. 


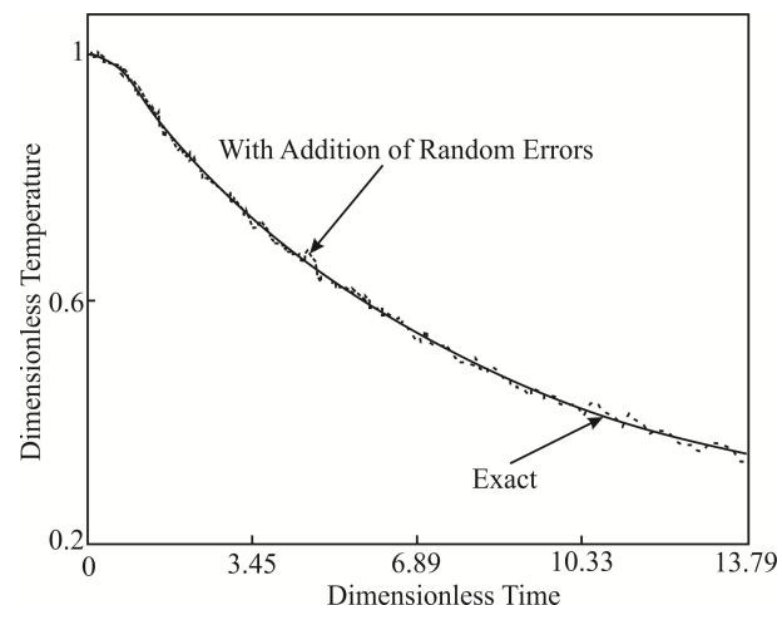

Figure 3.Dimensionless Simulated Measurements in the Inner Pipe

Different functional forms, including those containing sharp corners and discontinuities that are the most difficult to be recovered by the inverse analysis, were used to generate the simulated measurements. The functions used in this work are illustrated in Figures 4-5.

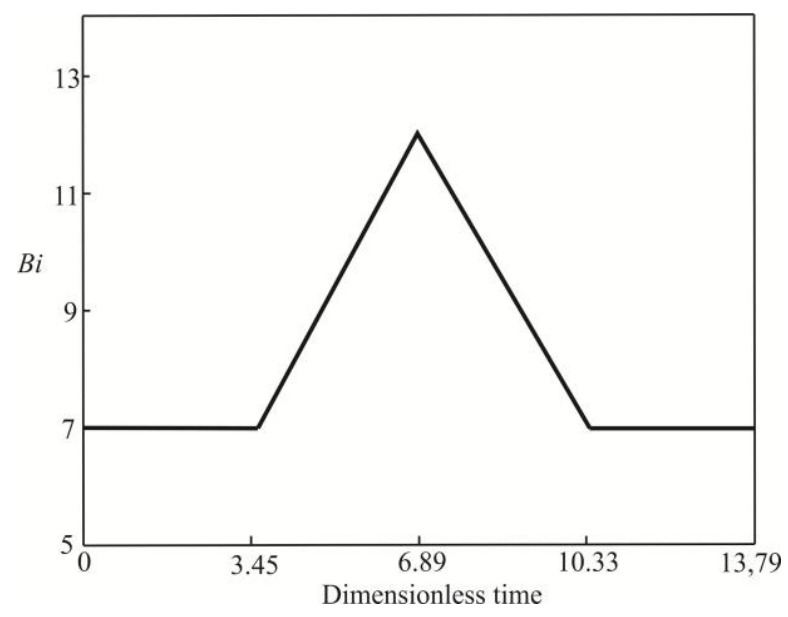

Figure 4. Function Used to Generate the Simulated Measurements (Triangular Function)

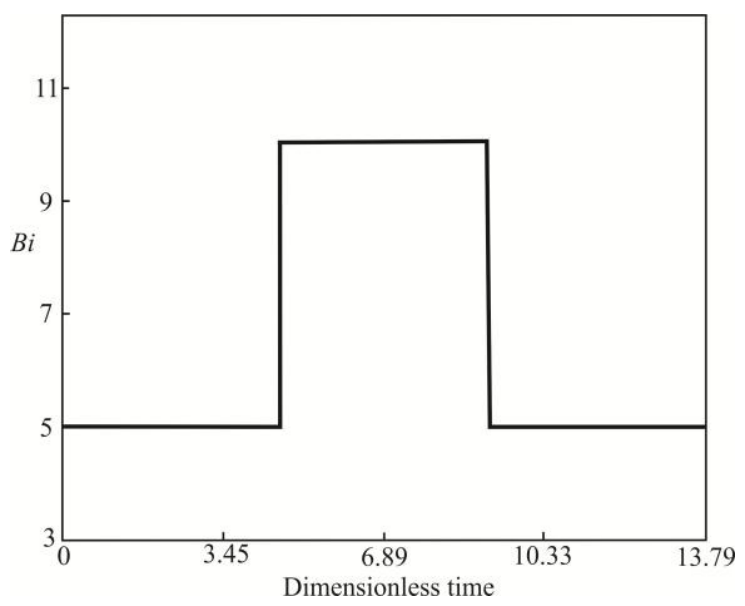

Figure 5. Function Used to Generate the Simulated Measurements (Step Function) 
The initial state used for the Markov chains were constant values $B i=9$ for the estimation of triangular function and $B i=7$ for the estimation of step function. A uniform jumping distribution was used to generate new samples in the Markov chain. The convective heat transfer coefficient $(h)$ on the external surface of the outer pipe for a practical case is approximately $2000 \mathrm{~W} / \mathrm{m}^{2} \mathrm{~K}$, based on Churchill-Bernstein's correlation [17], which corresponds to $B i=7,65$.

Figures 6-7 present the exact, estimated means and 99\% confidence intervals for the triangular and step variations of the heat transfer coefficient, respectively. These Figures show that excellent estimates can be obtained for the unknown function.

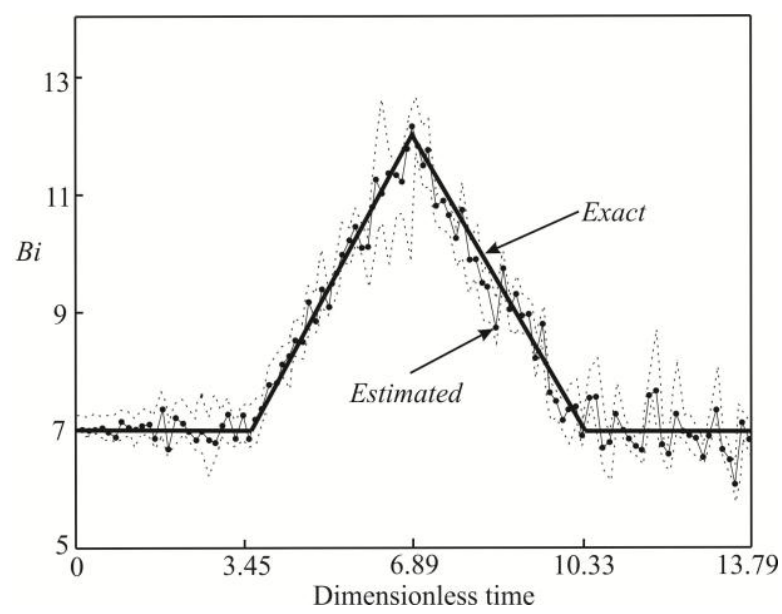

Figure 6.Estimation of Biot number values (triangular function)

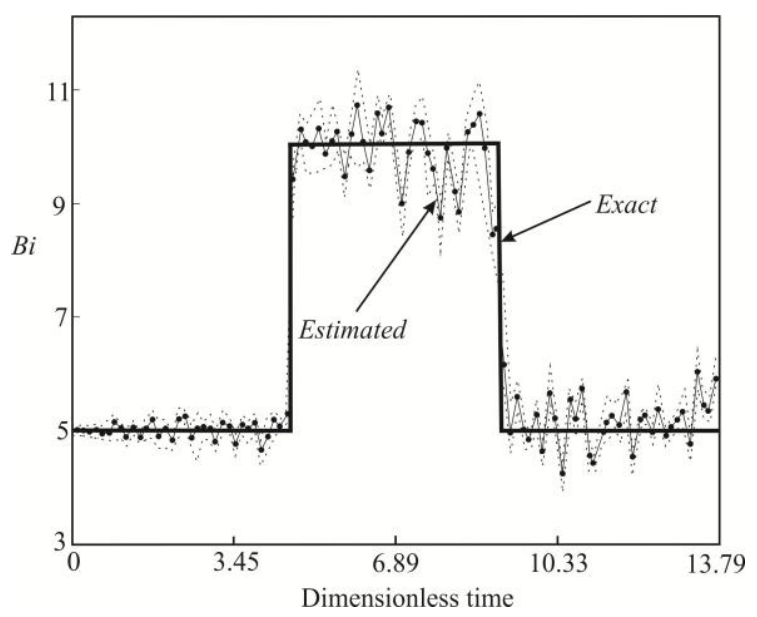

Figure 7.Estimation of Biot number values (step function)

Figures 8-9 illustrate the states of the Markov chains for the Biot number values at four different times, for the triangular and step variations, respectively. The Markov chains exhibit convergence in both cases after 10000 iterations and the acceptance ratio was around of $50 \%$. The expected values of the Biot numbers are also presented in these figures. 


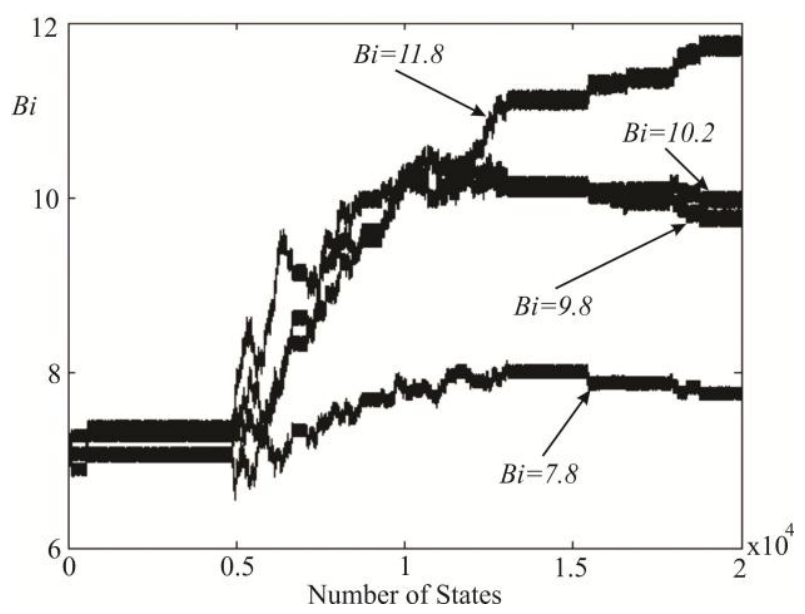

Figure 8.States of the Markov chains for four Biot number values corresponding to different times for a triangular function

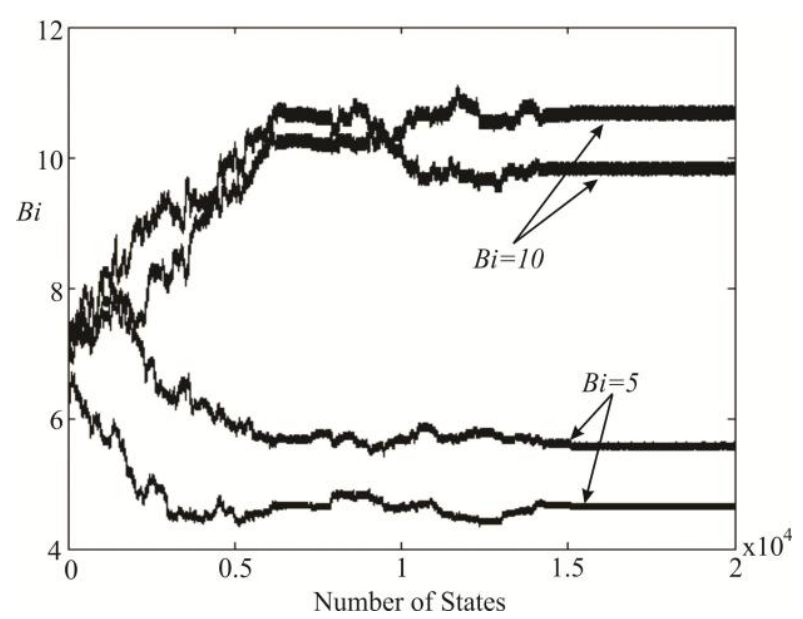

Figure 9.States of the Markov chains for four Biot number values corresponding to different times for a step function

The effects of the standard deviations of the measurement errors on the estimation of the Biot number, are examined below by considering $\sigma=0.005 \boldsymbol{\theta}_{\max }$ and $\sigma=0.05 \boldsymbol{\theta}_{\max }$, which correspond to $0.4^{\circ} \mathrm{C}$ and $4^{\circ} \mathrm{C}$, respectively.

Figures 10 and 11 present the exact and estimated means for the Biot numbers, as well as their $99 \%$ confidence intervals, for the triangular and step variations, respectively, with measurements of standard deviation $\sigma=0.005 \boldsymbol{\theta}_{\max }$. Similar results are presented in figures 12 and 13 for measurements of standard deviation $\sigma=0.05 \boldsymbol{\theta}_{\max }$. These figures show that excellent estimates could be obtained with low noise measurements. At the same time, we notice that the present solution approach was capable of accurately recovering the unknown function with a non-informative prior, even for quite large uncertainties in the measurements. 


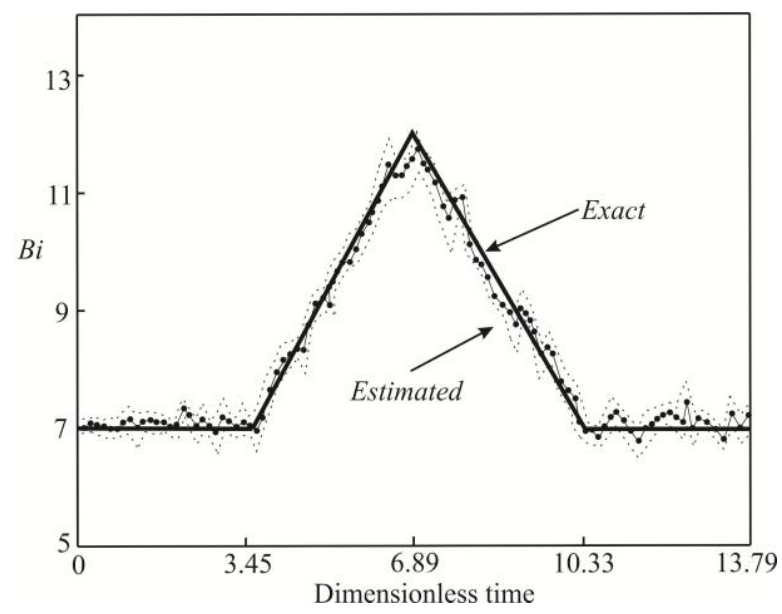

Figure 10.Estimation of Biot number values (triangular function) for $\sigma=0.005 \boldsymbol{\theta}_{\max }$

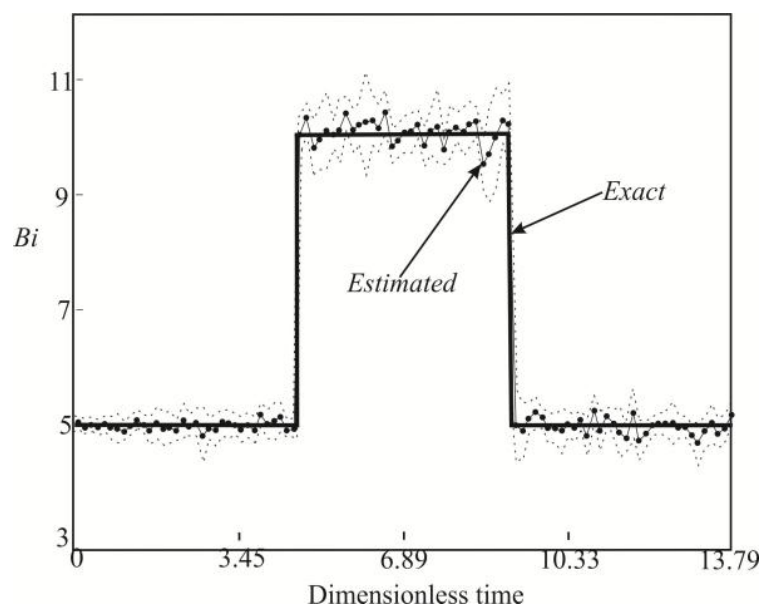

Figure 11.Estimation of Biot number values (step function) for $\sigma=0.005 \boldsymbol{\theta}_{\max }$

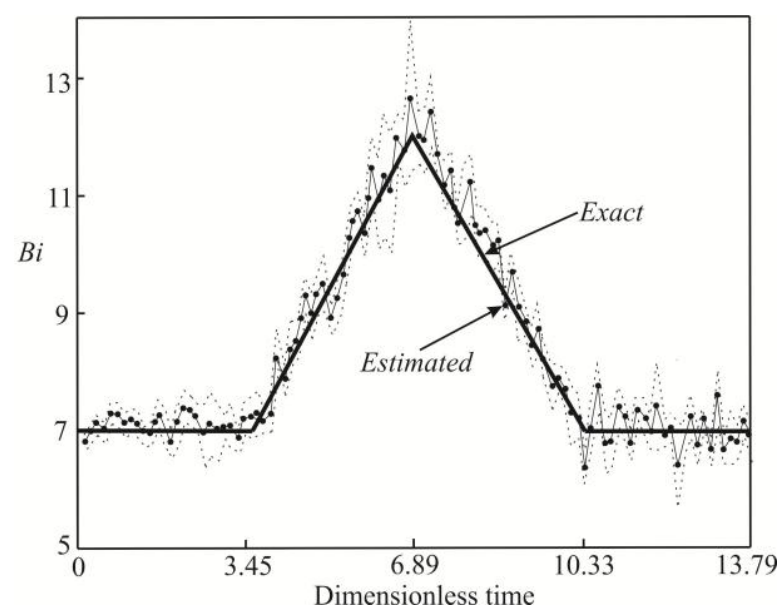

Figure 12.Estimation of Biot number values (triangular function) for $\sigma=0.05 \boldsymbol{\theta}_{\max }$ 


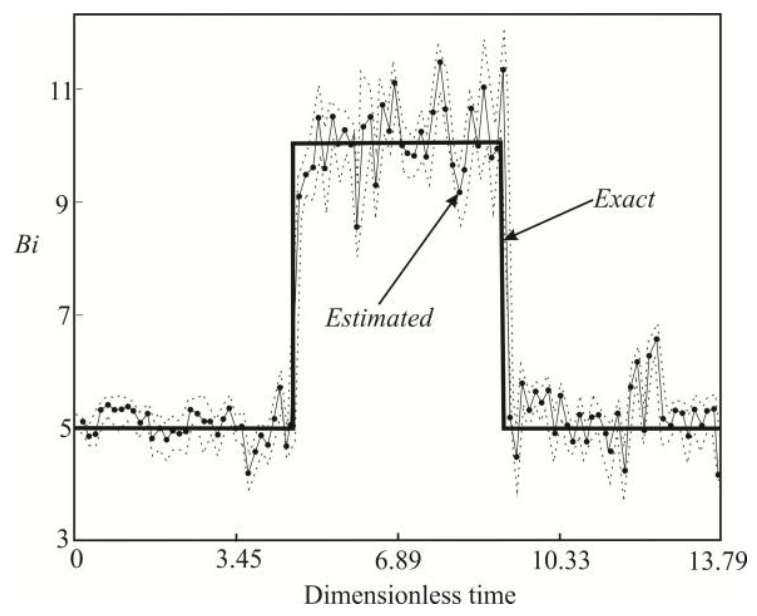

Figure 13.Estimation of Biot number values (step function) for $\sigma=0.05 \boldsymbol{\theta}_{\max }$

\section{CONCLUSIONS}

The inverse problem considered in this work dealt with the estimation of the convective heat transfer coefficient in pipe-in-pipe systems from temperature measurements containing random errors. The measurements were supposed to be taken at the external surface of the inner pipe. The inverse problem was solved with the Markov Chain Monte Carlo method, by using a non-informative smoothness prior for the unknown functions. Results obtained with simulated measurements for two different transient variations of the heat transfer coefficient, involving sharp corners and discontinuities, show that the MCMC method was capable of providing accurate and stable estimates for the unknown function. Furthermore, the method was very robust with respect to the measurement errors.

\section{Acknowledgements}

This work was supported by CNPq, CAPES and FAPERJ.

\section{REFERENCES}

[1] Flávio L. V. Vianna, Helcio R. B. Orlande and George S. Dulikravich. "Temperature field prediction of a multilayered composite pipeline based on the particle filter method.'Proceedings of the 14th International Heat Transfer Conference IHTC-14, Washington D.C., USA, August 8-13, 2010.

[2] Flávio L. V. Vianna, Helcio R. B. Orlande and George S. Dulikravich. "Optimal heating control to prevent solid deposits in pipelines.”V European Conference on Computational Fluid Dynamics ECCOMAS CFD 2010.

[3] R. M. T. Camargo, M. A. L. Gonçalves, J. R. T. Montesanti, C. A. B. R. Cardoso, and K. Minami, A Perspective View of Flow Assurance in Deepwater Fields in Brazil, OTC 16687, Offshore Technology Conference, May 3-6, Houston, Texas, USA (2004). 
[4] Beck, J. V. and Arnold, K. J., "Parameter Estimation in Engineering and Science", Wiley Interscience, New York. 1977.

[5] Kaipio, J. e Somersalo, E., "Statistical and Computational Inverse Problems", Applied Mathematical Sciences 160, Springer-Verlag, 2004.

[6] Calvetti, D., Somersalo, E.," Introduction to Bayesian Scientific Computing", Springer, New York. 2007.

[7] Tan, S., Fox, C., and Nicholls, G., "Inverse Problems", Course Notes for Physics 707, University of Auckland. 2006.

[8] Winkler, R., "An Introduction to Bayesian Inference and Decision", Probabilistic Publishing, Gainsville. 2003.

[9] Lee, P. M., “Bayesian Statistics”, Oxford University Press, London. 2004.

[10] Gamerman, D. and Lopes, H.F., "Markov Chain Monte Carlo: Stochastic Simulation for Bayesian Inference", Chapman \& Hall/CRC, 2nd edition, Boca Raton, 2006.

[11] Helcio R. B. Orlande, Marcelo José Colaço, Carolina P. N. Cotta, Gilmar Guimarães, ValerioLuiz Borges. "Problemas inversos em Transferência de calor". Sociedade Brasileira de Matemática Aplicada e Computacional. São Carlos - SP, Brasil 2011.

[12] Helcio R. B. Orlande. "Inverse problems in heat transfer", New trends on solution methodologies and applications Proceedings of the 14th International Heat Transfer Conference IHTC-14 Washington D.C., USA. August 8-13, 2010.

[13] Bolstad, William M. "Understanding Computational Bayesian Statistics", John Wiley 2010.

[14] Kaipio, J., Fox, C., "The Bayesian Framework for Inverse Problems in Heat Transfer", Heat Transfer Eng., 2010.

[15] Helcio R. B. Orlande, O. Fudym, D. Maillet e R. Cotta, "Thermal Measurements and Inverse Techniques", CRC Press, Boca Raton, 2011.

[16] Su, J. and Cerqueira, D.R., "Simulation of Transient Heat Transfer in Multilayered Composite Pipeline", Proceeding of OMAE01, 20nd International Conference on Offshore Mechanics and Arctic Engineering, Rio de Janeiro, Brazil. June 3-8, 2001.

[17] Incropera, F. P., Dewitt, D.P., 2006, "Fundamentals of Heat and Mass Transfer", Jonh Wiley and Sons. 